\title{
PAPER
}

\section{Four-vector description of the photon-in-a-box problem}

To cite this article: J Güémez and M Fiolhais 2019 Eur. J. Phys. 40025601

View the article online for updates and enhancements.

\section{IOP ebooks}

Bringing you innovative digital publishing with leading voices

to create your essential collection of books in STEM research.

Start exploring the collection - download the first chapter of every title for free. 


\title{
Four-vector description of the photon-in-a- box problem
}

\author{
J Güémez ${ }^{1}$ and $\mathbf{M}$ Fiolhais ${ }^{2,3}$ (1) \\ ${ }^{1}$ Department of Applied Physics, University of Cantabria, E-39005 Santander, Spain \\ ${ }^{2}$ Department of Physics and CFisUC, University of Coimbra, P-3004-516 Coimbra, \\ Portugal \\ E-mail: guemezj@unican.es and tmanuel@uc.pt
}

Received 4 October 2018, revised 8 November 2018

Accepted for publication 27 November 2018

Published 10 January 2019

\begin{abstract}
The emission/absorption of a photon and the launch/capture of a massive particle in special relativity Einstein-like boxes are studied, using the fourvector formalism. The study demands the introduction of the centre-of-inertia concept, the relativistic equivalent of the classical centre-of-mass of a system. Then, using Einstein's principle of the inertia of the energy and the conservation of energy and momentum, we show explicitly, in both cases, that the centre-of-inertia velocity does not change during the processes that take place inside the boxes. We argue that the centre-of-inertia is a useful concept in special relativity as counterpart of the centre-of-mass, and we suggest that it should be explored in the classroom to stress the similarities and the differences between the classical and the relativistic descriptions of mechanical systems.
\end{abstract}

Keywords: relativity, inertia of the energy, Einstein photon-in-a-box

\section{Introduction}

In this paper we study a variant of the 1906 Einstein's photon-in-a-box [1] and an interesting modification of it. Actually, here, we use the French's version of the Einstein's photon-in-abox [2], consisting of two unconnected parallel walls floating in outer space. A photon, in its original form, or a massive object, in our modified version, is emitted/launched from one wall and absorbed/capture by the other one.

Historically, this kind of gedankenexperiments [3], put forward by Einstein [4], played a crucial role in the formulation of the theory of special relativity. In particular, they contributed

3 Author to whom any correspondence should be addressed. 


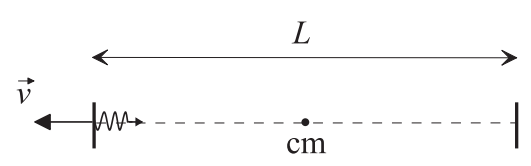

Figure 1. Emission of a photon by the left wall, with the centre-of-mass of the system $(\mathrm{cm})$ in the middle of the walls.

to establish the so-called principle of the inertia of the energy, an additional postulate of special relativity.

Our goal is to present a covariant description of the processes in the boxes, using the four-vector formalism, by assuming the principle of the inertia of energy [5]. In each process there is conservation of linear momentum and energy. Hence, the use of momentum-energy four-vectors is rather obvious, but, interesting enough, we are not aware of such treatments for the Einstein's photon-in-a-box in the literature. We note that the system we are going to study should not be confused with the 'Einstein's box', a much later (1930) famous thought experiment which played a major role in the Einstein-Bohr debates on quantum physics.

Before the covariant approach, it is worthwhile to briefly present the naïve approach. Let us consider two (unconnected) walls of mass $M$, which are at rest and separated by a distance $L$ - the French's simplified box. A photon (frequency $\nu$ ) is emitted from the left wall and absorbed, after a while, by the right wall. The process is shown in figure 1, where the recoil velocity of the left wall after emission, $\vec{v}$, is represented.

The energy of the emitted photon is $h \nu$ and its linear momentum is $h \nu / c$. Conservation of the linear momentum implies that the left wall moves to the left with linear momentum $-M v$, so

$$
-M v+\frac{h \nu}{c}=0 \Rightarrow v=\frac{h \nu}{M c}
$$

On the other hand, since there are no external forces acting upon the system, the centreof-mass velocity, initially zero, does not change. The centre-of-mass position is

$$
x_{\mathrm{cm}, \mathrm{i}}=\frac{M \times 0+M \times L}{2 M}=\frac{L}{2}
$$

and, in order to impose that the centre-of-mass remains stationary when the photon is absorbed by the right wall, a certain mass, $m$, has to be added to that wall. Since the left wall moves to the left a distance $v t$, where $t=L / c$ is the time elapsed from emission to absorption of the photon, the position of the centre-of-mass, when the photon is absorbed, is given by

$$
x_{\mathrm{cm}, \mathrm{f}}=\frac{-\frac{M L h \nu}{M c^{2}}+(M+m) L}{2 M} .
$$

From the condition $x_{\mathrm{cm}, \mathrm{f}}=x_{\mathrm{cm}, \mathrm{i}}$, one obtains

$$
m=c^{-2} h \nu
$$

and the conclusion is that the absorbed photon contributes to the right wall inertia as its energy divided by $c^{2}$.

It is clear that the above reasoning, though yielding a result that is considered correct, contains a number of inconsistencies [6]. For example, the mass $m$ is not included in the inertia of the left wall [7], the conservation of the energy is not taken into account, the origin of the final internal kinetic energy is unclear, etc. It is also worth mentioning that the description presented above is neither Galileo nor Lorentz covariant, similarly to the original 
description of the photoelectric effect [8]. In general, Einstein, in his analysis of these mental experiments, often uses a semi-classical approach, with relativistic and non-relativistic concepts in the same discussion. This is why the original analyses of Einstein were criticised, though everybody agrees that his final conclusion, the inertia of the energy, is correct [9].

The modern view, is that the principle of the inertia of the energy [10] is to be regarded as an additional hypothesis in the special relativity theory and not a consequence of it [11]. It states that the whole energy content of a system in its zero-momentum frame, say its internal energy, $E_{0}$, contributes to the system's inertia, $\mathcal{M}$, as $\mathcal{M}=c^{-2} E_{0}$. The zero-momentum frame is sometimes referred to as the centre-of-mass [12] or centre-of-inertia frame.

This paper is organised as follows. In section 2 we introduce the momentum-energy fourvectors for the relativistic description of an extended mechanical system and define its centreof-inertia velocity and coordinate. In section 3 the emission and absorption of the photon-in-abox is studied, using the four-vector formalism. In section 4 we carry on a similar study, now for a box with launching from one wall and subsequent capture by the other wall (inelastic collision) of a massive particle. Our conclusions, where we stress the usefulness of the centreof-inertia concept in relativity, are drawn in section 5 .

\section{Relativistic centre-of-inertia}

In relativity, the conservation of energy and of the linear momentum is better dealt with using four-vectors [13]. Our convention is that the momentum-energy four-vector of a particle is

$$
\mathrm{P}=\left(\begin{array}{c}
p_{0} \\
p_{1} \\
p_{2} \\
p_{3}
\end{array}\right)=\left(\begin{array}{c}
E / c \\
p_{x} \\
p_{y} \\
p_{z}
\end{array}\right),
$$

where the first (zeroth) component is the energy divided by $c$ and the remaining three component are the Cartesian components of the linear momentum.

In classical physics, for systems with several objects (such as a ball, a block, etc), the mass of the system is the sum of the masses of their different objects, and the concept of centre-of-mass is important and useful. The position and the velocity of the centre-of-mass provide information that characterises the motion of the system as a whole.

In relativity, let us represent by $\vec{P}_{\text {tot }}$ the total linear momentum of a system (sum of all linear momenta of its components) and by $E_{\text {tot }}$ its total energy (sum of all energies identified in the system, including binding energies, excitation energies, the internal kinetic energy, etc), in a given inertial reference frame, $S$. The centre-of-inertia velocity of that system, the relativistic equivalent of the centre-of-mass velocity (we prefer centre-of-inertia [14], 'ci', instead of centre-of-mass, to emphasise the energy contribution to the inertia of the system) in $S$ is

$$
\vec{v}_{\mathrm{ci}}=\frac{\vec{P}_{\mathrm{tot}}}{c^{-2} E_{\mathrm{tot}}} .
$$

We should mention that the term centre-of-energy, used by Boyer [15], is equivalent to centre-of-inertia as used in this paper.

Let's simplify the discussion since the $y$ and $z$ components always vanish in the problems treated in this article (we will only deal with one-dimensional motions). Hence, hereafter we represent a four-vector by just its two non-zero components. Therefore, a momentum-energy four-vector for a system in $1+1$ dimensions, is simply expressed by 


$$
\mathrm{P}_{\mathrm{tot}}=\left(\begin{array}{c}
E_{\mathrm{tot}} / c \\
P_{\mathrm{tot}}
\end{array}\right)
$$

It is worth checking that the centre-of-inertia velocity, as defined by (6) behaves indeed as a velocity, under Lorentz transformations. For the one-dimensional motion, (6) reduces to

$$
v_{\mathrm{ci}}=\frac{P_{\mathrm{tot}}}{c^{-2} E_{\mathrm{tot}}} .
$$

Consider now a reference frame $S^{\prime}$ moving with velocity $V$ in the positive $x$ direction (frame in standard configuration with respect to $S$ ). In this frame, the momentum-energy fourvector is $P^{\prime \mu}=\mathcal{L}_{\nu}^{\mu} P^{\nu}$ with the Lorentz transformation matrix given by

$$
\left(\mathcal{L}_{\nu}^{\mu}\right)=\left(\begin{array}{cc}
\gamma & -\beta \gamma \\
-\beta \gamma & \gamma
\end{array}\right),
$$

where $\beta=V / c$ and $\gamma=\left(1-\beta^{2}\right)^{-1 / 2}$. One obtains

$$
\mathrm{P}_{\mathrm{tot}}^{\prime}=\left(\begin{array}{c}
E_{\mathrm{tot}}^{\prime} / c \\
P_{\mathrm{tot}}^{\prime}
\end{array}\right)=\left(\begin{array}{c}
\gamma\left(E_{\mathrm{tot}}-\beta P_{\mathrm{tot}} c\right) / c \\
\gamma\left(-\beta E_{\mathrm{tot}}+P_{\mathrm{tot}} c\right) / c
\end{array}\right) .
$$

In the new reference frame, the centre-of-inertia velocity is given by

$$
v_{\mathrm{ci}}^{\prime}=\frac{P_{\mathrm{tot}}^{\prime}}{c^{-2} E_{\mathrm{tot}}^{\prime}},
$$

or, using (10)

$$
v_{\mathrm{ci}}^{\prime}=\frac{-\beta E_{\mathrm{tot}}+c P_{\mathrm{tot}}}{E_{\mathrm{tot}} / c-\beta P_{\mathrm{tot}}} .
$$

From equation (8) that defines $v_{\text {ci }}$ we can finally write the centre-of-inertia velocity in $S^{\prime}$ as

$$
v_{\mathrm{ci}}^{\prime}=\frac{v_{\mathrm{ci}}-V}{1-v_{\mathrm{ci}} V / c^{2}},
$$

which is the appropriate velocity transformation under a Lorentz boost.

An extended object has a certain internal energy, which is given by

$$
E_{0}=\sum_{j} \gamma_{j} m_{j} c^{2}+U
$$

where $m_{j}$ is the rest mass of the $j$ th elementary particle that forms the object, and $\gamma_{j}$ is the usual relativistic factor

$$
\gamma_{j}=\frac{1}{\sqrt{1-\left(v_{j} / c\right)^{2}}},
$$

where $v_{j}$ is the modulus of velocity of that particle $j$ in the reference frame in which the object is at rest as a whole (zero-momentum or centre-of-inertia frame). In (14), $U$ is the total binding energy.

The inertia of the extended object, $\mathcal{M}$, is assumed to be [16]

$$
\mathcal{M}=c^{-2} E_{0}
$$

as already mentioned in the Introduction. It may depend on the temperature, on the chemical composition, $\xi$, on the angular velocity [17], $\omega, \mathcal{M}=\mathcal{M}(T, \xi, \omega)$, etc. 


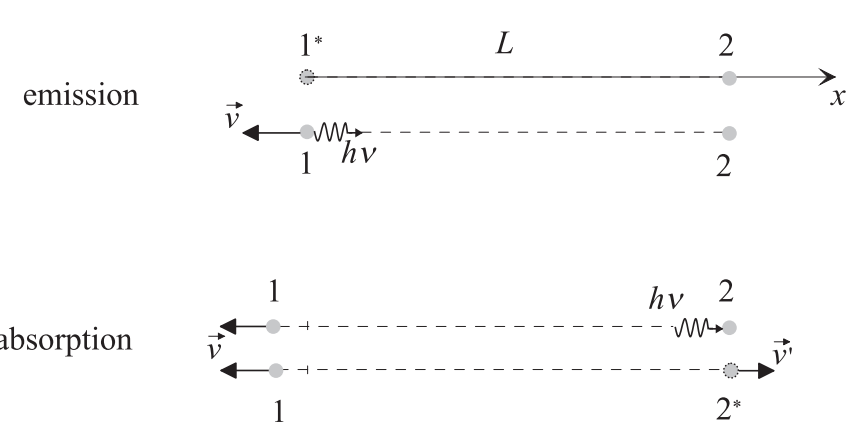

Figure 2. Emission and absorption of a photon in the box.

Let us consider a system composed of several objects (a wall, a ball, a spring, etc), each of which with inertia $\mathcal{M}_{k}$ (as defined above), and moving as a whole with velocity $\vec{v}_{k}$ (in the lab reference frame). The energy of the object is $E_{k}=\gamma_{k} \mathcal{M}_{k} c^{2}$, where $\gamma_{k}$ is the relativistic factor for the velocity $v_{k}$. For the one-dimensional motion the centre-of-inertia velocity is given by

$$
v_{\mathrm{ci}}=\frac{\sum_{k} E_{k} c^{-2} v_{k}}{E_{\mathrm{tot}} c^{-2}},
$$

where $k$ runs over all objects and interactions contained in the system.

The centre-of-inertia position is defined by [18]

$$
x_{\mathrm{ci}}=\frac{\sum_{k} E_{k} c^{-2} x_{k}}{E_{\mathrm{tot}} c^{-2}}
$$

(similarly for the other coordinates, $y_{\mathrm{ci}}$ and $z_{\mathrm{ci}}$, in $1+3$ dimensions).

\section{Einstein's photon-in-a-box}

We consider French's simplified version of the Einstein photon-in-a-box: two unconnected walls in outer space, perpendicular to the $x$ axis, are at rest in the $S$ reference frame (the lab frame). The inertia of each wall is $\mathcal{M}=c^{-2} E_{0}$, where $E_{0}$ is the wall internal energy, as discussed in the previous section. The two walls are separated by the distance $L$, as measured in the reference frame $S$ and, therefore, the associated total energy of the system is $2 E_{0}$ and the centre-of-inertia is at position $L / 2$.

However, the left wall is slightly different from the right one, because it contains an excited atom, ready to emit a photon. The detailed description of the emission and absorption of light is not relevant for the discussion and it is even worth stressing that it was not known when these famous gedankenexperiments were put forward by Einstein.

\subsection{Emission}

We can represent the left wall by a point-like element, as figure 2 shows. Initially, the total energy of the left wall $\left(1^{*}\right)$ is $\mathcal{M} c^{2}+\epsilon$, where $\epsilon$ stands for the excitation energy of the excited atom as measure in the lab frame. The inertia of the right wall is $\mathcal{M}$. The emission of the photon is accompanied by a recoil of the left wall (1), which acquires a velocity $\vec{v}$, as measured in the lab frame. In the same reference frame, the energy of the photon is $h \nu$. The momentum-energy four-vectors associated with the left wall (initial and final states) and with 
the right wall (no change during emission) are

$$
\mathrm{P}_{1 \mathrm{i}}=\left(\begin{array}{c}
\mathcal{M} c+\epsilon / c \\
0
\end{array}\right), \quad \mathrm{P}_{1 \mathrm{f}}=\left(\begin{array}{c}
\gamma \mathcal{M} c \\
-\gamma \mathcal{M} v
\end{array}\right), \quad \mathrm{P}_{2}=\left(\begin{array}{c}
\mathcal{M} c \\
0
\end{array}\right),
$$

and, for the emitted photon

$$
P_{\mathrm{ph}}=\left(\begin{array}{l}
h \nu / c \\
h \nu / c
\end{array}\right)
$$

The position and the velocity of the centre-of-inertia before the emission, using equations (18) and (17), are

$$
\begin{aligned}
& x_{\mathrm{ci}}=\frac{\left(\mathcal{M}+c^{-2} \epsilon\right) \times 0+\mathcal{M} \times L}{2 \mathcal{M}+c^{-2} \epsilon}=\frac{\mathcal{M} L}{2 \mathcal{M}+c^{-2} \epsilon}<\frac{L}{2}, \\
& v_{\mathrm{ci}}=\frac{\left(\mathcal{M}+c^{-2} \epsilon\right) \times 0+\mathcal{M} \times 0}{2 \mathcal{M}+c^{-2} \epsilon}=0 .
\end{aligned}
$$

Note that the excitation energy contributes to the inertia, hence the centre-of-inertia is not located symetrically with respect to the walls.

The conservation of the energy and of the linear momentum in the emission process can be expressed simultaneously, using four-vectors: $\mathrm{P}_{1 \mathrm{f}}-\mathrm{P}_{\mathrm{li}}+\mathrm{P}_{\mathrm{ph}}=0$, or, explicitly

$$
\left(\begin{array}{c}
\gamma \mathcal{M} c \\
-\gamma \mathcal{M} v
\end{array}\right)-\left(\begin{array}{c}
\mathcal{M} c+\epsilon / c \\
0
\end{array}\right)+\left(\begin{array}{l}
h \nu / c \\
h \nu / c
\end{array}\right)=\left(\begin{array}{l}
0 \\
0
\end{array}\right)
$$

leading to

$$
\left\{\begin{array}{l}
h \nu=\epsilon-(\gamma-1) \mathcal{M} c^{2} \\
h \nu=\gamma \mathcal{M} c \nu
\end{array}\right.
$$

These equations allow us to obtain the two unknowns, namely, the recoil velocity of the left wall and the energy of the emitted photon. By dividing term by term these two equations, one obtains the velocity. Introducing the parameter

$$
\alpha=1+\frac{\epsilon}{\mathcal{M} c^{2}}
$$

one arrives at

$$
\frac{v}{c}=\frac{\alpha^{2}-1}{\alpha^{2}+1}
$$

Then, the first equation in (24), for instance, gives the energy of the photon with $\gamma$ obtained from (26). That expression shows that $h \nu<\epsilon$ : part of the excitation energy, $\epsilon$, goes into kinetic energy of the left wall.

It is interesting to explicitly calculate the velocity and the position of the centre-of-inertia after emission, using the velocities and coordinates of the objects of the system. For the centre-of-inertia velocity one has

$$
v_{\mathrm{ci}}=\frac{-\gamma \mathcal{M} v+\frac{h \nu}{c^{2}} c+\mathcal{M} \times 0}{\gamma \mathcal{M}+\mathcal{M}+\frac{h \nu}{c^{2}}}=\frac{c^{-1}(h \nu-\gamma \mathcal{M} c v)}{\gamma \mathcal{M}+\mathcal{M}+\frac{h \nu}{c^{2}}}=0,
$$

where use has been made of the second equation in (24). For the centre-of-inertia position, with $0 \leqslant t \leqslant L / c$ 


$$
x_{\mathrm{ci}}=\frac{\gamma \mathcal{M} \times(-v t)+\frac{h \nu}{c^{2}} \times c t+\mathcal{M} L}{\gamma \mathcal{M}+\frac{h \nu}{c^{2}}+\mathcal{M}}=\frac{\mathcal{M} L}{2 \mathcal{M}+c^{-2} \epsilon},
$$

where we have used, in the numerator and in the denominator, both expressions (24). This is in agreement with equation (18).

Another important check of our results is the analysis of the classical limit, corresponding to $\epsilon \ll \mathcal{M} c^{2}$. In this case, from (25) and (26), the velocity is given by

$$
\frac{v}{c} \simeq \frac{\epsilon}{\mathcal{M} c^{2}} \ll 1
$$

Taking the approximation $\gamma \simeq 1+\frac{1}{2} \frac{v^{2}}{c^{2}}$ in the first equation (24), the energy of the photon becomes

$$
h \nu \simeq \epsilon-\frac{1}{2} \mathcal{M} v^{2}
$$

where the second term on the right-hand side is the classical kinetic energy of the left wall.

\subsection{Absorption}

When the emitted photon hits the right wall, it is absorbed (does not matter in which way) and the inertia of the wall becomes $\mathcal{M}+c^{-2} \epsilon^{\prime}$, as it starts moving to the right with velocity $v^{\prime}$. The process is represented in figure 2 (bottom), immediately before and after absorption. The momentum-energy four-vectors associated with the right wall (initial and final states) are

$$
\mathrm{P}_{2 \mathrm{i}}=\left(\begin{array}{c}
\mathcal{M} c \\
0
\end{array}\right), \quad \mathrm{P}_{2 \mathrm{f}}=\left(\begin{array}{c}
\gamma^{\prime}\left(\mathcal{M}+c^{-2} \epsilon^{\prime}\right) c \\
\gamma^{\prime}\left(\mathcal{M}+c^{-2} \epsilon^{\prime}\right) v^{\prime}
\end{array}\right)
$$

where $\gamma^{\prime}$ stands for the relativistic factor associated with velocity $v^{\prime}$. For the photon, the momentum-energy four-vector is given by (20) and for the left wall (which keeps its motion to the left) it is given by the second expression in (19).

The momentum-energy conservation, expressed using these four-vectors, is

$$
\left(\begin{array}{c}
\mathcal{M} c \\
0
\end{array}\right)+\left(\begin{array}{l}
h \nu / c \\
h \nu / c
\end{array}\right)=\left(\begin{array}{c}
\gamma^{\prime}\left(\mathcal{M}+c^{-2} \epsilon^{\prime}\right) c \\
\gamma^{\prime}\left(\mathcal{M}+c^{-2} \epsilon^{\prime}\right) v^{\prime}
\end{array}\right)
$$

leading to

$$
\left\{\begin{array}{l}
h \nu+\mathcal{M} c^{2}=\gamma^{\prime}\left(\mathcal{M}+c^{-2} \epsilon^{\prime}\right) c^{2} \\
h \nu=\gamma^{\prime}\left(\mathcal{M}+c^{-2} \epsilon^{\prime}\right) v^{\prime} c
\end{array}\right.
$$

From these equations we can obtain the two unknowns: the velocity of the right wall, $v^{\prime}$, and the energy acquired by that wall after photon absorption, $\epsilon^{\prime}$. For the former one readily obtains

$$
\frac{v^{\prime}}{c}=\frac{\alpha^{2}-1}{\alpha^{2}+2 \alpha-1}
$$

where $\alpha$ was introduced in (25). Comparing with (26), one concludes that, as expected, $v^{\prime}<v$. On the other hand, from (33) one obtains 


$$
\epsilon^{\prime}=\left(\sqrt{\alpha+1-\frac{1}{\alpha}}-1\right) \mathcal{M} c^{2} .
$$

From the first equations in (24) and (33), we can write ( $v$ and $v^{\prime}$ have already been obtained)

$$
\epsilon-\epsilon^{\prime}=(\gamma-1) \mathcal{M} c^{2}+\left(\gamma^{\prime}-1\right)\left(\mathcal{M}+c^{-2} \epsilon^{\prime}\right) c^{2},
$$

i.e. $\epsilon^{\prime} \leqslant \epsilon$, as expected: the final internal kinetic energy equals the difference $\epsilon-\epsilon^{\prime}$.

The centre-of-inertia velocity is given by

$$
v_{\mathrm{ci}}=\frac{-\gamma \mathcal{M} v+\gamma^{\prime}\left(\mathcal{M}+c^{-2} \epsilon^{\prime}\right) v^{\prime}}{\gamma \mathcal{M}+\gamma^{\prime}\left(\mathcal{M}+c^{-2} \epsilon^{\prime}\right)} .
$$

Using both the second equations in (24) and (33) one concludes that $v_{\mathrm{ci}}=0$, as it should be. In line with this result, the position of the centre-of-inertia does not change. Noting that, when the photon hits the right wall, the left wall is located at $-v L / c$, so one has $(t>0)$

$$
x_{\mathrm{ci}}=\frac{-\gamma M v\left(\frac{L}{c}+t\right)+\gamma^{\prime}\left(\mathcal{M}+c^{-2} \epsilon^{\prime}\right)\left(L+v^{\prime} t\right)}{\gamma \mathcal{M}+\gamma^{\prime}\left(\mathcal{M}+c^{-2} \epsilon^{\prime}\right)} .
$$

Using equations (24) and (33) one arrives at

$$
x_{\mathrm{ci}}=v_{\mathrm{ci}} t=\frac{-\frac{h \nu}{c^{2}} L+\frac{h \nu}{c^{2}} L+\mathcal{M} L}{2 \mathcal{M}+c^{-2} \epsilon}
$$

which is nothing but equation (18). This result explicitly confirms that the centre-of-inertia does not move.

The next step in our description of the process is the study of the classical limit, $h \nu \ll \mathcal{M} c^{2}$. Since $\alpha^{2}-1 \simeq \frac{2 \epsilon}{\mathcal{M} c^{2}}$, from (34)

$$
\frac{v^{\prime}}{c} \simeq \epsilon \mathcal{M} c^{2}=\frac{v}{c}
$$

On the other hand, for the absorbed energy, one obtains

$$
\epsilon^{\prime} \simeq \frac{\epsilon}{\mathcal{M} c^{2}} \mathcal{M} c^{2}=\epsilon
$$

Using the approximation $\gamma^{\prime} \simeq 1+\frac{1}{2} \frac{v^{\prime 2}}{c^{2}}$ in the first equation in (33) one obtains

$$
h \nu \simeq \epsilon^{\prime}+\frac{1}{2} \mathcal{M} v^{\prime 2}
$$

showing that the energy of the photon goes partly to kinetic energy of the right wall and partly to increase its internal energy. Combining this result with (30) one arrives at the following expression (see also equation (36)):

$$
\epsilon-\epsilon^{\prime} \simeq \frac{1}{2} \mathcal{M}\left(v^{2}+v^{\prime 2}\right),
$$

whose interpretation is rather obvious.

We can apply the conservation of the energy and the linear momentum to the initial and final wall states, $\mathrm{P}_{1 \mathrm{i}}+\mathrm{P}_{2 \mathrm{i}}=\mathrm{P}_{1 \mathrm{f}}+\mathrm{P}_{2 \mathrm{f}}$. Explicitly

$$
\left(\begin{array}{c}
2 \mathcal{M} c+\epsilon / c \\
0
\end{array}\right)=\left(\begin{array}{c}
\gamma \mathcal{M} c+\gamma^{\prime}\left(\mathcal{M}+c^{-2} \epsilon^{\prime}\right) c \\
-\gamma \mathcal{M} v+\gamma^{\prime}\left(\mathcal{M}+c^{-2} \epsilon^{\prime}\right) v^{\prime}
\end{array}\right)
$$




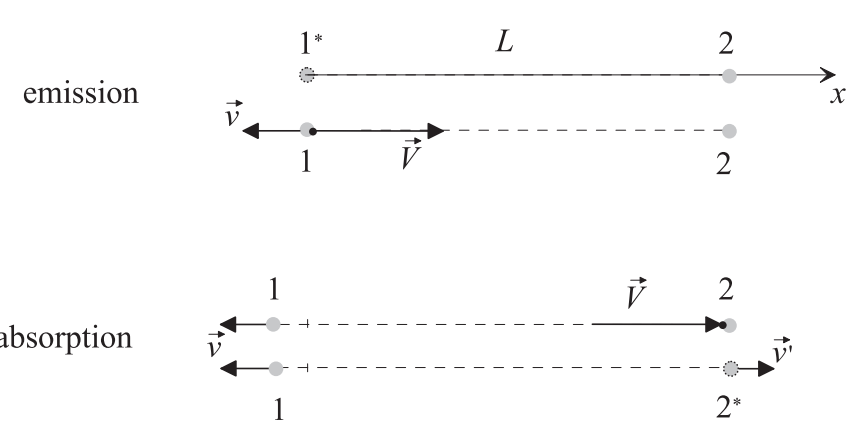

Figure 3. Box with the launching and capture of a massive particle.

One obtains two equations for three unknowns $\left(v, v^{\prime}\right.$ and $\left.\epsilon^{\prime}\right)$. Actually, one needs the detailed description of the emission of the absorption, as done in this section, to obtain the three quantities.

\section{Box with a massive particle}

The example to be studied next shows quite a few similarities with the previous one. However, the differences are noticeable enough to make worthwhile the study of the Einstein's photon-in-a-box but with the launching of a massive object. Again, we have two walls, as described in the previous section, but now the left wall contains an additional object of mass $m$, a ball, which, at some point, will be launched. The system walls + ball stores an internal e.g. elastic potential energy, $\epsilon$. We may imagine that this energy is due to a massless spring [19] that is released, launching the ball in the $x$ direction with velocity $V$. Such a launching process is depicted at the top of figure 3.

\subsection{Particle launching}

The right wall, 2, remains at rest during this process. For the walls, the momentum-energy four-vectors pertinent for the emission process are

$$
\mathrm{P}_{1 \mathrm{i}}=\left(\begin{array}{c}
\mathcal{M} c+m c+\epsilon / c \\
0
\end{array}\right), \quad \mathrm{P}_{1 \mathrm{f}}=\left(\begin{array}{c}
\gamma \mathcal{M} c \\
-\gamma \mathcal{M} v
\end{array}\right), \quad \mathrm{P}_{2}=\left(\begin{array}{c}
\mathcal{M} c \\
0
\end{array}\right)
$$

where $\mathrm{i} / \mathrm{f}$ refer to before/after emission, and

$$
\mathrm{P}_{\mathrm{b}}=\left(\begin{array}{c}
\gamma_{V} m c \\
\gamma_{V} m V
\end{array}\right)
$$

is the momentum-energy four-vector for the emitted ball. Here, $\gamma$ stands for the relativistic factor associated with velocity $v$ (left wall), and similarly for $\gamma_{V}$ which stands for the ball whose velocity is $V$.

The initial centre-of-inertia velocity is zero:

$$
v_{\mathrm{ci}}=0
$$

since all parts of the system (walls and ball) are at rest in the lab reference frame. The centreof-inertia position is 


$$
x_{\mathrm{ci}}=\frac{\mathcal{M} L}{2 \mathcal{M}+m+c^{-2} \epsilon}<\frac{L}{2} .
$$

The conservation of the energy and of the linear momentum in the emission process can be expressed simultaneously, using four-vectors: $P_{1 f}-P_{1 i}+P_{b}=0$, or, explicitly

$$
\left(\begin{array}{c}
\gamma \mathcal{M} c \\
-\gamma \mathcal{M} v
\end{array}\right)-\left(\begin{array}{c}
\mathcal{M} c+m c+\epsilon / c \\
0
\end{array}\right)+\left(\begin{array}{c}
\gamma_{v} m c \\
\gamma_{v} m V
\end{array}\right)=\left(\begin{array}{l}
0 \\
0
\end{array}\right),
$$

leading to

$$
\left\{\begin{array}{l}
\gamma_{V} m=\left(\mathcal{M}+m+c^{-2} \epsilon\right)-\gamma \mathcal{M} \\
\gamma_{V} m V=\gamma M v
\end{array}\right.
$$

These equations allow us to obtain both $v$ and $V$. As previously (see (26)), one obtains

$$
\frac{v}{c}=\frac{\alpha^{2}-1}{\alpha^{2}+1},
$$

where the parameter $\alpha$ is now redefined and it is given by

$$
\alpha=1+\frac{m c^{2}+\epsilon}{\mathcal{M} c^{2}} .
$$

For the ball's velocity, one obtains

$$
\frac{V}{c}=\frac{\mu}{\sqrt{1+\mu^{2}}}
$$

where we have introduced a new parameter:

$$
\mu=\frac{\alpha^{2}-1}{2 \alpha} \frac{\mathcal{M}}{m} .
$$

The centre-of-inertia velocity, immediately after the launching of the ball is

$$
v_{\mathrm{ci}}=\frac{-\gamma \mathcal{M} v+\gamma_{v} m V}{\gamma \mathcal{M}+\gamma_{v} m+\mathcal{M}}=0,
$$

where use has been made of the second equation in (50). The centre-of-inertia position is

$$
x_{\mathrm{ci}}=\frac{\gamma \mathcal{M} \times(-v t)+\gamma_{V} m \times V t+\mathcal{M} L}{\gamma \mathcal{M}+\gamma_{v} m+\mathcal{M}} .
$$

Using both expressions (50), one readily obtains (48): the centre-of-inertia of the system does not move due to the emission process.

Let us now check the classical limit, corresponding to $\left(m c^{2}+\epsilon\right) \ll \mathcal{M} c^{2}$ and for which $v^{\prime}$ and also $V$ are assumed to be small. From (51) and (52):

$$
\frac{v}{c} \simeq \frac{m c^{2}+\epsilon}{\mathcal{M} c^{2}} \ll 1 .
$$

Using the approximations $\gamma \simeq 1-\frac{v^{2}}{2 c^{2}}$ and $\gamma_{V} \simeq 1-\frac{V^{2}}{2 c^{2}}$ in the first equation (50) one arrives at

$$
\epsilon \simeq \frac{1}{2} \mathcal{M} v^{2}+\frac{1}{2} m V^{2}
$$




\subsection{Capture by inelastic collision}

After travelling the distance $L$ with velocity $V$, the ball impacts the right wall (meanwhile the left wall kept moving to the left with velocity $-v$ ). We assume that collision to be inelastic with the ball adhering to the right wall, becoming part of it. The right wall starts moving right, with velocity $v^{\prime}$ and increases its inertia by $m+c^{-2} \epsilon^{\prime}$, where $\epsilon^{\prime}$ stands for the increase of internal energy experienced by the wall + ball in the process [20] (the detailed process how this energy increase occurs is not relevant for the discussion). The process is described in figure 3 (bottom). Expressing the momentum-energy conservation using four-vectors, one may write

$$
\left(\begin{array}{c}
\mathcal{M} c \\
0
\end{array}\right)+\left(\begin{array}{c}
\gamma_{V} m c \\
\gamma_{V} m V
\end{array}\right)=\left(\begin{array}{c}
\gamma^{\prime}\left(\mathcal{M}+m+c^{-2} \epsilon^{\prime}\right) c \\
\gamma^{\prime}\left(\mathcal{M}+m+c^{-2} \epsilon^{\prime}\right) v^{\prime}
\end{array}\right),
$$

( $\gamma^{\prime}$ is the relativistic factor for $v^{\prime}$ ) leading to

$$
\left\{\begin{array}{l}
\mathcal{M}+\gamma_{V} m=\gamma^{\prime}\left(\mathcal{M}+m+c^{-2} \epsilon^{\prime}\right) \\
\gamma_{V} m V=\gamma^{\prime}\left(\mathcal{M}+m+c^{-2} \epsilon^{\prime}\right) v^{\prime}
\end{array}\right.
$$

From these equations we can obtain the two unknowns: the velocity of the right wall, $v^{\prime}$, and the energy acquired by that wall after the collision, $\epsilon^{\prime}$.

To obtain the velocity, one divides term by term equations (60) to obtain

$$
\frac{v^{\prime}}{c}=\frac{\gamma_{V} m V}{\mathcal{M} c+\gamma_{V} m c}
$$

Using now (53) and recognising that $\gamma_{V} \frac{V}{c}=\mu$, one arrives at

$$
\frac{v^{\prime}}{c}=\frac{m \mu}{\mathcal{M}+m \sqrt{1+\mu^{2}}}
$$

The explicit dependence on $\epsilon, m$ and $\mathcal{M}$ can be obtained using (52) and (54). The energy $\epsilon^{\prime}$ can now be obtained inserting the expression for $v^{\prime}$ in one of the equations (60). These calculations are straightforward but the final expression is lengthy. From the first equations in (50) and (60) we obtain ( $v$ and $v^{\prime}$ have already been obtained)

$$
\epsilon-\epsilon^{\prime}=(\gamma-1) \mathcal{M} c^{2}+\left(\gamma^{\prime}-1\right)\left(\mathcal{M}+m+c^{-2} \epsilon^{\prime}\right) c^{2},
$$

hence $\epsilon^{\prime}<\epsilon$. The final internal kinetic energy equals $\epsilon-\epsilon^{\prime}$.

We may proceed with the explicit confirmation of the values for the centre-of-inertia velocity and position. The centre-of-inertia velocity is given by

$$
v_{\mathrm{ci}}=\frac{-\gamma M v+\gamma^{\prime}\left(M+m+c^{-2} \epsilon^{\prime}\right) v^{\prime}}{\gamma M+\gamma^{\prime}\left(M+m+c^{-2} \epsilon^{\prime}\right)}=0,
$$

where we have used the second equations in (50) and in (60). Since $x_{\mathrm{ci}}(t)=x_{\mathrm{ci}}(0)+v_{\mathrm{ci}} t$, the centre-of-inertia remains at the same position during the whole process. But it is instructive to explicitly show that $x_{\mathrm{ci}}(t)=x_{\mathrm{ci}}(0)$. The centre-of-inertia position after the collision is

$$
x_{\mathrm{ci}}=\frac{-\gamma \mathcal{M} v\left(\frac{L}{V}+t\right)+\gamma^{\prime}\left(\mathcal{M}+m+c^{-2} \epsilon^{\prime}\right)\left(L+v^{\prime} t\right)}{\gamma M+\gamma^{\prime}\left(M+m+c^{-2} \epsilon^{\prime}\right)} .
$$

Using expressions (50) and (60), in the numerator and in the denominator, one finally arrives at the result expressed by equation (48). 
Next, let us look at the classical limit. From the first expression in (60) one may write

$$
\frac{1}{2} m V^{2} \simeq \epsilon^{\prime}+\frac{1}{2}(\mathcal{M}+m) v^{\prime 2}
$$

The left-hand side in (66) is the kinetic energy of the ball before the impact. On the right-hand side one has the sum of the internal energy increase and the kinetic energy of the right wall plus ball.

Combining equations (58) and (66), one finally obtains (see also equation (63))

$$
\epsilon-\epsilon^{\prime} \simeq \frac{1}{2} \mathcal{M} v^{2}+\frac{1}{2}(\mathcal{M}+m) v^{\prime 2}
$$

\section{Conclusions}

The centre-of-inertia (or centre-of-energy) velocity, the relativistic counterpart of the classical centre-of-mass velocity, was defined as the total linear momentum of a system, in a certain reference frame, divided by the total inertia of that system, measured in the same reference frame. In the absence of external forces acting upon the system, the centre-of-inertia velocity remains unchanged. Using the four-vector formalism, we explicitly showed this result in the context of the famous Einstein's photon-in-a-box, which was important in the development of the idea of the equivalence between mass and energy. Actually, using the French's modified version of the special relativity Einstein's box, we explicitly showed that the centre-of-inertia of the system remains unchanged (if it is initially at rest) when emission/absorption of a photon or of a massive particle takes place in the boxes.

We claim that the centre-of-inertia is a useful concept in special relativity, as the centreof-mass is in classical mechanics, and suggest that such concept should be explored in the classroom to stress the differences and the similarities between the classical and the relativistic descriptions of mechanical systems.

\section{ORCID iDs}

M Fiolhais (iD https://orcid.org/0000-0002-6636-9957

\section{References}

[1] Antippa A F 1976 Inertia of energy and the liberated photon Am. J. Phys. 44 841-4

[2] French A P 1968 Special Relativity (London: Chapman and Hall) p 24

[3] Rohrlich F 1990 An elementary derivation of $E=m c^{2}$ Am. J. Phys. 58 348-9

[4] Kantor W 1954 Inertia of energy Am. J. Phys. 22 528-41

[5] Hecht E 2011 How Einstein confirmed $E_{0}=m c^{2}$ Am. J. Phys. 79 591-600

[6] Hecht R 2012 How Einstein discovered $E_{0}=m c^{2}$ Phys. Teach. 50 91-4

[7] Feenberg E 1960 Inertia of energy Am. J. Phys. 28 565-6

[8] Güémez J and Fiolhais M 2018 Relativistic description of the photoelectric effect Am. J. Phys. 86 $825-30$

[9] Rigney C J and Biser R H 1966 Note on a famous derivation of $E=m c^{2}$ Am. J. Phys. 34623

[10] Güémez J 2010 An undergraduate exercise in the first law of relativistic thermodynamics Eur. $J$. Phys. 31 1209-32

[11] Hecht E 2009 Einstein on mass and energy Am. J. Phys. 77 799-806

[12] Rosser W G V 1991 Introductory Special Relativity (London: Taylor and Francis) pp 150-1 
[13] Okun L B 2009 Mass versus relativistic and rest masses Am. J. Phys. 77 430-1

[14] Ferraro R 2007 Einstein's Space-Time. An Introduction to Special and General Relativity (Berlin: Springer) p 153

[15] Boyer T H 2005 Illustrations of the relativistic conservation law for the center of energy Am. J. Phys. 73 953-61

[16] Hecht E 2011 On defining mass Phys. Teach. 49 40-4

[17] Güémez J, Fiolhais M and Fernández L A 2017 Relativistic rotation dynamics-formalism and examples Europhys. Lett. 11910001

[18] Sandin T R 1991 In defense of relativistic mass Am. J. Phys. 59 1032-6

[19] Dib C 2013 Mass as a form of energy in a simple example Phys. Teach. 51 546-8

[20] Güémez J, Fiolhais M and Fernández L A 2016 Relativistic mechanical-thermodynamical formalism-description of inelastic collisions Eur. J. Phys. 37015602 